\title{
Resistência à penetração de um solo em recuperação sob sistemas agrosilvopastoris ${ }^{1}$
}

\author{
Fabiana da S. de Campos ${ }^{2} \&$ Marlene C. Alves ${ }^{2}$
}

\section{RESUMO}

Na Fazenda de Ensino e Pesquisa da Universidade Estadual Paulista (UNESP) Campus de Ilha Solteira, localizada em Selvíria, no Estado de Mato Grosso do Sul, conduziu-se um estudo com o objetivo de investigar a resistência de um solo à penetração, solo este altamente degradado pela construção de uma Usina Hidrelétrica. A área experimental tem aproximadamente $15552 \mathrm{~m}^{2}$ cultivado com Pinus. Na determinação da resistência à penetração foi utilizado o penetrográfo Penetrographer ${ }^{\text {Pat }}$ SC-60 nas profundidades de 0-0,05, 0,05-0,10, 0,10-0,20 e 0,20-0,40 m. O delineamento experimental utilizado foi o inteiramente casualizado, com cinco tratamentos e cinco repetições. Os dados obtidos foram submetidos à análise de variância e as médias comparadas pelo teste Tukey, a 5\% de probabilidade. Os tratamentos foram constituídos dos seguintes usos e manejos do solo: (T1) área em estado avançado de degradação; (T2) área em regeneração cultivada com Pinus há 13 anos; (T3) área em regeneração cultivada com adubos verdes/pastagem há 11 anos; (T4) área degradada com regeneração natural e área com vegetação natural de cerrado (Testemunha). Os resultados obtidos indicam que o sistema com regeneração em que foram utilizados o Pinus e os nichos com regeneração natural, foram os que mais se aproximaram da condição natural de cerrado. Com exceção da área natural (cerrado) na camada de 0,200,40 m, todas as demais áreas mostraram alta resistência à penetração, o que representa sérias limitações ao crescimento das raízes.

Palavras-chave: manejo do solo, degradação do solo, retenção de água

\section{Resistance to penetration of a soil in reclamation under agrosilvo-pastoral system}

\begin{abstract}
In the Research and Teaching Farm of the State University of São Paulo (UNESP) - Campus of Ilha Solteira, located in Selviria, in Mato Grosso do Sul State, a study was conducted with the objective of investigating the resistance to penetration of a highly degraded soil due to construction of a Hydroelectric Power Plant. The experimental design was a completely randomized, with five treatments and five replications. The treatments were constituted of the following uses and managements: area in advanced state of degradation; area under regeneration cultivated 13 years with Pinus; area under regeneration cultivated 11 years with green manure and pasture; degraded area with natural regeneration; and area with natural vegetation (savanna). The system where the area was under regeneration with Pinus cultivation and the niches with natural regeneration were the closest to natural condition (savanna). Except for the natural area (savanna), all others presented a higher resistance to penetration in the layer of $0.20-0.40 \mathrm{~m}$, which represents serious limitations for growth of plant roots.
\end{abstract}

Key words: soil management, soil degradation, water retention

${ }^{1}$ Parte do trabalho de graduação do primeiro autor, apresentada à UNESP, financiado pelo CNPq/PIBIC

${ }^{2}$ FE/IS/UNESP. CP 31, CEP 15385-000, Ilha Solteira, SP. Fone: (18) 37431143, ramal 1742. Fax: 37431176. E-mail: fscampos@aluno.feis.unesp.br, mcalves@agr.feis.unesp.br 


\section{INTRODUÇÃO}

Os conceitos ambientais atualmente discutidos, baseiamse nas teorias do desenvolvimento sustentável, que consideram o aperfeiçoamento das práticas e da melhoria do desempenho dos empreendimentos, através de efetividade e da garantia de atendimento às exigências ambientais, entre as quais se inclui a recuperação de áreas degradadas (Fornasari Filho et al., 1994).

De acordo com a EMBRAPA (2001) para qualquer grande obra de engenharia é conveniente a movimentação de solo para os trabalhos de terraplanagem e construção civil, expondo camadas de solo que podem resultar em processos erosivos superficiais ou laminares, em sulcos e até voçorocas. Esses processos, além de causarem o assoreamento das linhas de drenagem podem, no seu estágio mais avançado, colocar em risco edificações e vias de acesso. Assim, muitas vezes a ocorrência de grandes áreas impermeabilizadas e/ou a sistematização de terrenos associados à falta e/ou deficiência de um sistema de drenagem das águas pluviais, pode causar a desestabilização, queda de taludes e o aparecimento de processos erosivos de grandes dimensões.

O problema de degradação da área em estudo foi gerado em conseqüência da construção da Usina Hidrelétrica de Ilha Solteira, SP. Ainda que a hidroeletricidade, como alternativa tecnológica para produção de energia, possa ser considerada ambientalmente mais vantajosa em relação a outras opções, por utilizar um recurso natural renovável e não poluente, a formação de reservatórios implica na ocorrência de diversos impactos ao ambiente, atingindo elementos físicos, biológicos e socioeconômicos (CESP, 1998).

É verdade que grande parte do impacto, tanto sobre o ambiente amtrópico quanto sobre o ambiente natural, é localizado no entorno das industrias, justificando-se o meio retorno de riquezas ou benefícios para estes locais. No aspecto físico, as empresas têm aumentado os cuidados ambientais devido às exigências mundiais, mas há muito para fazer. A legislação privilegia a burocracia, enquanto a técnica deve mais aos programas de certificados ambientais aos quais, felizmente, a maioria das grandes companhias hidrelétricas vêm aderindo.

As áreas remanescentes da construção de usinas hidrelétricas geralmente provocam degradação nos ecossistemas envolvidos, pois promovem eliminação, juntamente com a vegetação, dos seus meios de regeneração bióticos, como o banco de sementes, banco de plântulas, chuvas de sementes e rebrota. As áreas apresentam, portanto, baixa resiliência, isto é, seu retorno ao estado anterior pode não ocorrer ou ser extremamente lento.

Para a recuperação, é preciso selecionar e identificar espécies aptas às novas condições edáficas e que, de forma rápida, acelere a estruturação e formação dos horizontes mais superficiais do solo (Carpanezzi et al., 1994). A adaptação e o desenvolvimento dessas espécies dependerão das condições físicas, químicas, biológicas e hídricas do solo e também das condições do microclima local.

Qualquer interferência visando à restauração de ecossis- temas, deve partir de dois pressupostos: de que exista um ecossistema original, em equilíbrio dinâmico, cujas características têm de ser respeitadas no processo de restauração como ideal a ser atingido, e de que existem instrumentos legais que regulamentam interferências sobre os recursos naturais.

A resistência do solo à penetração das raízes é uma das propriedades físicas que influenciam diretamente o crescimento das raízes e da parte aérea das plantas. Vários autores utilizaram a resistência do solo à penetração para a avaliação dos efeitos dos sistemas de manejo do solo sobre o ambiente radicular (Benghough \& Mullins, 1990; Tormena \& Roloff, 1996).

Daniel et al. (1994) estudando as mudanças nas características da resistência do solo sob diferentes manejos, concluíram que ferramentas que provocam um grau maior de mobilidade do solo, como arado de disco, grade aradora e a enxada rotativa, proporcionaram valores mais elevados de resistência do solo à penetração, indicando a presença de camadas compactadas; eles comentam, ainda, que a compactação ou a dureza do solo está intimamente ligada à umidade e que uma possível compactação pode ser mascarada pela elevada umidade do solo, no momento da amostragem.

Considerando-se a influência dos diferentes tipos de uso e manejo sobre as propriedades do solo objetivou-se, com o presente trabalho, estudar a resistência à penetração de um Latossolo Vermelho de uma área que teve 8,6 m de camada de solo extraída, devido à construção da Usina Hidrelétrica de Ilha Solteira, SP; sob cinco condições de uso e manejo do solo. A mesma está em processo de recuperação com sistemas agrosilvopastoris.

\section{MATERIAL E MÉTODOS}

O trabalho foi conduzido na Fazenda de Ensino e Pesquisa da Faculdade de Engenharia de Ilha Solteira - UNESP, localizada no município de Selvíria, MS, apresentando, como coordenadas geográficas, $51^{\circ} 22^{\prime}$ de longitude Oeste de Greenwich e $20^{\circ} 22^{\prime}$ de latitude Sul, com altitude de $335 \mathrm{~m}$. O relevo é caracterizado como moderadamente plano e ondulado. A vegetação original encontrada na área foi descrita como cerrado. A classificação do solo é Latossolo Vermelho Distrófico textura média - LVd (Demattê, 1980; EMBRAPA, 1999). Na Tabela 1 apresentam-se as caracterizações da granulometria, da densidade e características químicas, nas camadas de 0-0,15 e de 0,15-0,30 m, do solo original (Demattê, 1980).

A precipitação e a temperatura média anual são de $1370 \mathrm{~mm}$ e $23,5^{\circ} \mathrm{C}$, respectivamente, e a umidade relativa do ar varia entre 70 e $80 \%$, média anual. A área estudada localiza-se próximo ao reservatório da Usina Hidrelétrica de Ilha Solteira e se trata de uma área que sofreu forte impacto ambiental quando, a partir da construçuão da Usina final da década de 60, se retirou uma camada de solo de $8,6 \mathrm{~m}$ para os trabalhos de terraplanagem e construção civil, expondo o subsolo da área de estudo. Desde o início 
Tabela 1. Granulometria, densidade e características químicas do solo original e decapitado, em duas camadas

\begin{tabular}{|c|c|c|c|c|c|}
\hline \multirow{3}{*}{ Granulometria } & & \multirow{3}{*}{$\begin{array}{c}\text { Original } \\
0-0,15\end{array}$} & \multicolumn{3}{|c|}{ Decapitado } \\
\hline & & & \multicolumn{2}{|c|}{ Profundidade } & \\
\hline & & & $0,15-0,30$ & $0-0,20$ & $0,20-0,40$ \\
\hline Argila & & 220 & 250 & 256 & 267 \\
\hline Silte & & 30 & 00 & 82 & 70 \\
\hline \multirow{3}{*}{ Areia } & Fina & 270 & 290 & & \\
\hline & Grossa & 480 & 460 & & \\
\hline & Total & 750 & 750 & 662 & 663 \\
\hline Densidade $\mathrm{kg} \mathrm{dm}^{-1}$ & & 1,5 & 1,4 & 1,79 & 1,90 \\
\hline $\mathrm{pH}$ & & 4,5 (KCl) & 4,2 (KCl) & 4,0 $\left(\mathrm{CaCl}_{2}\right)$ & 4,2 $\left(\mathrm{CaCl}_{2}\right)$ \\
\hline $\mathrm{P}\left(\mathrm{mmol}_{\mathrm{c}}\right)$ & & 0,1 & 0,2 & 1,6 & 1,4 \\
\hline $\mathrm{Ca}\left(\mathrm{mmol}_{\mathrm{c}}\right)$ & & 6,5 & 0,5 & 1,1 & 0,6 \\
\hline $\mathrm{Mg}\left(\mathrm{mmol}_{\mathrm{c}}\right)$ & & 5,2 & 0,6 & 0,7 & 0,0 \\
\hline $\mathrm{K}\left(\mathrm{mmol}_{\mathrm{c}}\right)$ & & 0,7 & 0,2 & 0,6 & 0,1 \\
\hline $\mathrm{S}\left(\mathrm{mmol}_{\mathrm{c}}\right)$ & & 12,4 & 1,3 & 3,1 & 4,2 \\
\hline $\mathrm{Al}\left(\mathrm{mmol}_{\mathrm{c}}\right)$ & & 22,0 & 7,0 & & \\
\hline $\mathrm{H}\left(\mathrm{mmol}_{\mathrm{c}}\right)$ & & 22,0 & 4,0 & & \\
\hline $\mathrm{T}\left(\mathrm{mmol}_{\mathrm{c}}\right)$ & & 56,4 & 12,3 & 18,2 & 17,3 \\
\hline V (\%) & & 22,0 & 10,6 & 12,9 & 6,4 \\
\hline $\mathrm{Al}(\%)$ & & 63,2 & 84,0 & & \\
\hline C (\%) & & 0,73 & 049 & & \\
\hline $\mathrm{HH}+\mathrm{Al}\left(\mathrm{mmol}_{\mathrm{c}}\right)$ & & & & 15,7 & 16,2 \\
\hline $\mathrm{SB}\left(\mathrm{mmol}_{\mathrm{c}}\right)$ & & & & 2,6 & 1,1 \\
\hline $\mathrm{M} 0\left(\mathrm{~g} \mathrm{dm}^{-3}\right)$ & & & & 1,93 & 1,6 \\
\hline
\end{tabular}

da década de 70 esta área, que reúne 700 ha, está, em sua maioria, sujeita à regeneração natural, sendo que algumas pesquisas têm sido desenvolvidas na tentativa de sua recuperação. Na Tabela 1 apresentam-se algumas características físicas e químicas, na camada de 0-0,20 e de 0,200,40 m, do solo decapitado.

O delineamento experimental utilizado foi o inteiramente casualizado, com cinco tratamentos e cinco repetições. Os tratamentos foram constituídos dos seguintes usos e manejos: área em estado avançado de degradação (AD); área em regeneração, cultivada com Pinus há 13 anos (AR + Pinus); área em regeneração cultivada com adubos verdes/pastagem há 11 anos $(\mathrm{AR}+\mathrm{AV}+\mathrm{P})$; área degradada com regeneração natural $(\mathrm{AD}+\mathrm{RN})$ e área com vegetação natural de cerrado (C). Cada parcela teve dimensão de $10 \mathrm{~m}$ de largura por $7 \mathrm{~m}$ de comprimento.

Avaliou-se a textura do solo pelo método da pipeta (EMBRAPA, 1997), nas profundidades de 0-0,05; 0,05-0,10; $0,10-0,20$ e $0,20-0,40 \mathrm{~m}$, sendo que as amostras deformadas foram secadas ao ar e passadas em peneira de $2 \mathrm{~mm}$ de malha, para que pudesse ser realizada a dispersão das partículas e a sedimentação. A resistência do solo à penetração foi avaliada nas mesmas profundidades que a sua textura, utilizando-se o penetrográfo Penetrographer Pat SC-60. A umidade gravimétrica foi determinada pelo método da pesagem (EMBRAPA, 1997) nas mesmas profundidades da avaliação da resistência à penetração.

Os resultados foram analisados efetuando-se a análise de variância e o teste de Tukey, para comparação de médias, a 5\% de probabilidade. Empregou-se o programa computacional SAS/STAT (1990) para análise dos dados.

\section{RESULTADOS E DISCUSSÃO}

Para a profundidade de 0-0,05 m (Tabela 2) na área em estado avançado de degradação (AD), os resultados de resistência à penetração foram maiores comparados ao cerrado, área de regeneração cultivada com Pinus (AR + Pinus) e área degradada com regeneração natural ( $\mathrm{AD}+\mathrm{RN})$, com maior resistência a penetração verificada para a profundidade de 0,20-0,40 m. Este comportamento na área em estado avançado de degradação é explicado pela ausência de plantas e cobertura morta, presença de camada compactada nessa profundidade e pelo menor conteúdo de água no solo.

Para a profundidade de 0,05-0,10 m a área em estado avançado de degradação também apresentou resistência à penetração maior, diferindo da área de cerrado e da degradada com regeneração natural.

Tabela 2. Valores médios* de resistência à penetração e umidade do solo, para os respectivos tratamentos, nas profundidades estudadas

\begin{tabular}{|c|c|c|c|c|}
\hline \multirow{2}{*}{ Tratamento } & \multicolumn{4}{|c|}{ Profundidade do Solo (m) } \\
\hline & $0-0,05$ & $0,05-0,10$ & $0,10-0,20$ & $0,20-0,40$ \\
\hline \multicolumn{5}{|c|}{ Resistência à Penetração do Solo - MPa } \\
\hline$A D$ & $5,92 \mathrm{~A}$ & $4,96 \mathrm{~A}$ & $4,61 \mathrm{AB}$ & $6,92 \mathrm{~A}$ \\
\hline $\mathrm{AR}+$ Pinus & $1,89 \mathrm{~B}$ & $3,01 \mathrm{ABC}$ & $3,09 \mathrm{AB}$ & $7,60 \mathrm{~A}$ \\
\hline$A R+A V+P$ & $2,72 A B$ & $3,70 \mathrm{AB}$ & $5,04 \mathrm{~A}$ & $6,24 \mathrm{~A}$ \\
\hline $\mathrm{AD}+\mathrm{RN}$ & $2,38 \mathrm{~B}$ & $2,10 \mathrm{BC}$ & $1,38 \mathrm{AB}$ & $4,78 \mathrm{~A}$ \\
\hline C & $0,72 \mathrm{~B}$ & $0,94 \mathrm{C}$ & $0,98 \mathrm{~B}$ & $1,26 \mathrm{~B}$ \\
\hline CV (\%) & 62,80 & 43,32 & 64,23 & 34,31 \\
\hline Média Geral & 2,72 & 2,94 & 3,02 & 5,36 \\
\hline DMS (5\%) & 3,23 & 2,41 & 3,66 & 3,47 \\
\hline \multicolumn{5}{|c|}{ Umidade do Solo $-\mathrm{m}^{3} \mathrm{~m}^{-3}$} \\
\hline$A D$ & $6,57 \mathrm{~B}$ & $8,65 \mathrm{~B}$ & $9,45 \mathrm{~A}$ & $9,88 \mathrm{~B}$ \\
\hline AR + Pinus & $10,34 \mathrm{AB}$ & $11,81 \mathrm{~A}$ & $12,34 \mathrm{~A}$ & $12,57 \mathrm{~A}$ \\
\hline$A R+A V+P$ & $9,59 \mathrm{AB}$ & $11,37 \mathrm{~A}$ & $11,98 \mathrm{~A}$ & $12,66 \mathrm{~A}$ \\
\hline$A D+R N$ & $11,90 \mathrm{~A}$ & $11,63 \mathrm{~A}$ & $13,62 \mathrm{~A}$ & $12,99 \mathrm{~A}$ \\
\hline C & $9,47 \mathrm{AB}$ & $9,73 \mathrm{AB}$ & $9,85 \mathrm{~A}$ & 9,47 B \\
\hline CV (\%) & 22,38 & 13,36 & 23,01 & 9,82 \\
\hline Média Geral & 9,57 & 10,64 & 11,45 & 11,51 \\
\hline DMS (5\%) & 4,05 & 2,68 & 4,98 & 2,14 \\
\hline
\end{tabular}

*Médias seguidas de letras iguais na coluna não diferem estatisticamente entre si, pelo teste de Tukey, a $5 \%$ de probabilidade

Também pela Tabela 1, observa-se que não houve efeitos significativos a $5 \%$ de probabilidade entre os tratamentos $\mathrm{AD}$; $\mathrm{AR}+\mathrm{AV}+\mathrm{P}$ e AD + RN para a profundidade de 0,20$0,40 \mathrm{~m}$, exceto para o tratamento $\mathrm{C}$, mesmo não detectando significância, os valores médios de resistência à penetração do solo, superou o tratamento testemunha (C).

Atentando-se para os dados de resistência, verificou-se que em todas as profundidades estudadas a área com vegetação natural de cerrado (C) foi aquela com menor valor de resistência, como esperado, devido ao fato desta área se apresentar com suas condições naturais. Segundo Hakansson et al. (1998) e Larson et al. (1994), uma das medidas para prevenir a compactação seria fazer a incorporação e manutenção da matéria orgânica, calagem e sistema de semeadura direta.

Analisando-se os valores de resistência à penetração obtidos para os tratamentos estudados, verificou-se que a área 
com estado avançado de degradação (AD) na profundidade de 0,00-0,05 m se encontra na classe de resistência do solo à penetração considerada alta, segundo Canarache (1990); isto significa que o solo está com sérias limitações ao crescimento das raízes. Este solo em condições naturais (cerrado) se apresenta sem limitação ao crescimento das raízes, pois a classe de resistência à penetração é muito baixa (<1,1 MPa). O autor sugeriu que valores acima de 2,5 MPa começam a restringir o pleno crescimento das raízes das plantas; portanto, de acordo com os resultados obtidos na área com vegetação natural de cerrado, em todas as profundidades estudadas as plantas não teriam restrições ao crescimento de suas raízes, ao passo que nos demais tratamentos haveria algumas limitações na superfície do solo $(0,00-0,10 \mathrm{~m})$ e sérias limitações de 0,10-0,40 m.

Com relação aos efeitos dos usos e manejos na recuperação do solo em estudo verificou-se que, na camada de 0,00-0,05 m, os mesmos estão interagindo de forma semelhante. As condições atuais quanto a resistência à penetração, são iguais, quando interpretadas estatisticamente, porém quanto a classificação de Canarache (1990), os tratamentos área em regeneração cultivada com Pinus (AR + Pinus) e área degradada com regeneração natural $(\mathrm{AD}+\mathrm{RN})$ se enquadram na classe baixa (1,1-2,5 MPa) e a área em regeneração cultivada com adubo verde/pastagem $(\mathrm{AR}+\mathrm{AV}+\mathrm{P})$ na classe média (2,6-5,0 MPa), respectivamente, com pouca limitação e algumas estipulações ao crescimento das raízes. Segundo Souza \& Alves (2003), analisando-se a resistência à penetração em um Latossolo Vermelho de cerrado, sob diferentes usos e manejos, verificou-se que os menores resultados de resistência à penetração foram observados nas áreas do cerrado e cultivo mínimo. Para o sistema de pastagem notou-se um pico de resistência à penetração na profundidade de 0,10-0,20 m, podendo esta compactação ser resultante de pisoteio excessivo promovido pelos animais durante o pastejo.

Na camada de 0,05-0,10 m o comportamento entre os tratamentos foi semelhante, notando-se apenas que a área em regeneração cultivada com adubo verde/pastagem se apresentou mais resistente que a área de cerrado. A área em regeneração cultivada com Pinus sai da classe baixa e vai para a classe média, com relação à limitação ao crescimento das raízes.

Para a camada de 0,10-0,20 m somente o tratamento área em regeneração cultivada com adubo verde/pastagem diferiu da condição natural (cerrado), porém não diferiu dos demais tratamentos. Com exceção das áreas natural e degradada com regeneração natural, que se classificaram com baixa resistência à penetração, os outros tratamentos se enquadraram com média resistência. Na camada de 0,20-0,40 m, todos os tratamentos se diferenciaram das condições naturais de cerrado e se classificaram com média a alta resistência à penetração.

Verificou-se que os tratamentos com regeneração natural e área com regeneração + Pinus foram os mais promissores quanto aos índices de resistência à penetração, relacionados a menores restrições ao crescimento radicular. Notou-se que o efeito da regeneração está na camada de 0,00-0,05 m, em virtude da influência da adição da matéria orgânica. Alves
(2001) obteve resultados idênticos, trabalhando com recuperação de solo em condições semelhantes às desta pesquisa.

Nas condições naturais, o solo estudado apresenta horizonte A com 0,15 m de espessura (Demattê, 1980), mediante os resultados verificados por Alves (2001); Silva et al. (2002) e Andrade Júnior (2004), o solo decapitado, em estudo com diferentes formas de recuperação, apresenta horizonte A formado novamente e com espessura de $0,10 \mathrm{~m}$.

Com base nos dados de umidade do solo expostos na Tabela 5, observa-se diferença significativa para os tratamentos nas profundidades de 0-0,05, 0,05-0,10 e 0,20-0,40 m, exceto para a profundidade de $0,10-0,20$ m revelou que não houve efeito significativo a $5 \%$ de probabilidade em relação aos tratamentos estudados.

Na profundidade de 0-0,05 m o tratamento AD, diferiu da área degradada com regeneração natural (AD + RN). A área em estado avançado de degradação (AD) apresentou menor valor de umidade do solo, pelo fato desta área se apresentar compactada, o que leva à baixa capacidade de infiltração de água e armazenamento. De acordo com Souza \& Alves (2003), a infiltração de água indica diferenças no comportamento hidrodinâmico do solo, em função da alteração de sua estrutura, considerando-se que a infiltração de água se relaciona com as condições físicas do solo, como estrutura, porosidade e ausência de camadas compactadas. Deduzse que nos solos estudados elas sofreram modificações acentuadas, em função do manejo. As maiores alterações foram encontradas no solo sob pastagem e seringueira, em função da elevada resistência do solo à penetração de água.

Para a profundidade de 0,05-0,10 m, observou-se diferenças significância entre $\mathrm{AD}$ e os tratamentos $\mathrm{AR}+\mathrm{AV}+\mathrm{P}$, $\mathrm{AD}+\mathrm{RN}$ e C. Nesta profundidade também se observou menor valor de umidade para a $\mathrm{AD}$, porém não diferindo da área com cerrado $(\mathrm{C})$. A alta resistência à penetração do solo da $\mathrm{AD}$, está relacionada também ao menor conteúdo de água neste tratamento, porém se ressalta a presença de degradação da estrutura (compactação), pois na área com vegetação natural (cerrado), a umidade não diferiu da área degradada e também se encontrava baixa; no entanto, a resistência à penetração estava muito baixa. O grau de umidade intervém, modificando a coesão entre as partículas do solo, que é maior no solo seco e decresce a medida em que a quantidade de água aumenta, provocando a separação das suas partículas (Klein et al., 1998).

Não houve diferença significativa entre os tratamentos para a profundidade de 0,10-0,20 m. Observou-se, no entanto, que na profundidade de $0,20-0,40 \mathrm{~m}$ os tratamentos (AD) e (C) não diferiram estatisticamente entre si mas, sim, dos demais tratamentos.

A determinação da umidade do solo no momento da avaliação da resistência à penetração, é fundamental para se realizar adequadamente a interpretação dos resultados encontrados. No estudo em questão, por exemplo, na camada de 0,10-0,20 m não ocorreu diferença entre os tratamentos; quanto à umidade do solo, porém, verificou-se diferença para a resistência à penetração, o que reforça a interpretação efetuada, de vez que as diferenças encontradas não estão sendo influenciadas pela umidade. 
Observando-se a Tabela 3, verificou-se diferença estatística entre os tratamentos em todas as profundidades estudadas, quando se analisou o conteúdo de argila e areia do solo e, para as profundidades de 0,00-0,05; 0,05-0,10 e 0,20-0,40 m constatou-se, quando analisada a argila, que a área com vegetação natural de cerrado (C) diferiu estatisticamente dos demais tratamentos, apresentando valor menor, mas para a profundidade de 0,10-0,20 m, o (C) não diferiu da (AD) mas, sim, estatisticamente dos demais tratamentos.

Tabela 3. Valores médios* dos conteúdos de argila, areia e silte $\left(\mathrm{g} \mathrm{kg}^{-1}\right)$ do solo estudado para os tratamentos e profundidades analisadas

\begin{tabular}{|c|c|c|c|c|}
\hline \multirow{2}{*}{ Tratamento } & \multicolumn{4}{|c|}{ Profundidade (m) } \\
\hline & $0,00-0,05$ & $0,05-0,10$ & $0,10-0,20$ & $0,20-0,40$ \\
\hline \multicolumn{5}{|c|}{ Argila } \\
\hline$A D$ & $256 \mathrm{~A}$ & $246 \mathrm{~A}$ & $266 \mathrm{AB}$ & $267 \mathrm{~A}$ \\
\hline $\mathrm{AR}+$ Pinus & $295 \mathrm{~A}$ & $294 \mathrm{~A}$ & $289 \mathrm{~A}$ & $283 \mathrm{~A}$ \\
\hline$A R+A V+P$ & $305 \mathrm{~A}$ & $300 \mathrm{~A}$ & $295 \mathrm{~A}$ & $285 \mathrm{~A}$ \\
\hline$A D+R N$ & $263 \mathrm{~A}$ & $250 \mathrm{~A}$ & $260 \mathrm{~A}$ & $270 \mathrm{~A}$ \\
\hline C & $130 \mathrm{~B}$ & $110 B$ & $150 \mathrm{~B}$ & $160 \mathrm{~B}$ \\
\hline \multicolumn{5}{|c|}{ Areia } \\
\hline$A D$ & $665 \mathrm{AB}$ & $665 \mathrm{BC}$ & $657 \mathrm{~B}$ & $663 \mathrm{~B}$ \\
\hline $\mathrm{AR}+$ Pinus & $571 \mathrm{~B}$ & $627 \mathrm{BC}$ & $575 B$ & $616 \mathrm{BC}$ \\
\hline$A R+A V+P$ & $596 \mathrm{~B}$ & $588 \mathrm{C}$ & $598 \mathrm{~B}$ & $593 \mathrm{C}$ \\
\hline$A D+R N$ & 652 B & 677 B & $590 \mathrm{~B}$ & $660 \mathrm{~B}$ \\
\hline C & $781 \mathrm{~A}$ & $790 \mathrm{~A}$ & $773 \mathrm{~A}$ & $797 \mathrm{~A}$ \\
\hline \multicolumn{5}{|c|}{ Silte } \\
\hline$A D$ & 79 B & $89 \mathrm{~A}$ & $77 \mathrm{~A}$ & $70 \mathrm{BC}$ \\
\hline $\mathrm{AR}+$ Pinus & $134 \mathrm{~A}$ & $79 \mathrm{~A}$ & $136 \mathrm{~A}$ & $101 \mathrm{AB}$ \\
\hline$A R+A V+P$ & $99 \mathrm{AB}$ & $112 \mathrm{~A}$ & $107 \mathrm{~A}$ & $122 \mathrm{~A}$ \\
\hline$A D+R N$ & 85 B & $73 \mathrm{~A}$ & $150 \mathrm{~A}$ & $70 \mathrm{BC}$ \\
\hline C & $89 \mathrm{~B}$ & $100 \mathrm{~A}$ & $77 \mathrm{~A}$ & $43 \mathrm{C}$ \\
\hline
\end{tabular}
a $5 \%$ de probabilidade

Notou-se, nesta mesma tabela que, para todas as profundidades estudadas, a área com vegetação natural de cerrado apresentou maior conteúdo de areia. Cavenage (1996) encontrou, na mesma área do estudo, os seguintes teores de areia para a área de Pinus: 633, 606 e $575 \mathrm{~g} \mathrm{~kg}^{-1}$ nas respectivas profundidades de $0-0,10,0,10-0,20$ e $0,20-0,40 \mathrm{~m}$, valores semelhantes aos verificados na pesquisa.

Na Tabela 3 consta que, para a profundidade de 0,00 0,05 m, a área degradada cultivada com Pinus (AR + Pinus) mostrou maior conteúdo de silte (133 $\left.\mathrm{g} \mathrm{kg}^{-1}\right)$; enfim, este tratamento não diferiu estatisticamente apenas da $\mathrm{AR}+\mathrm{AV}+\mathrm{P}$, visto que para as profundidades de 0,05-0,10 e 0,10-0,20 m, os tratamentos não diferiram entre si. Na profundidade de 0,20-0,40 m, a área degradada cultivada com adubo verde + pastagem indicou maior valor de silte, porém não diferiu da área em regeneração cultivada com Pinus. Cavenage (1996), analisando a textura do solo na mesma área em estudo, constatou valores de silte menores que os apresentados neste trabalho para a área com Pinus, que foram 44, 58 e $43 \mathrm{~g} \mathrm{~kg}^{-1}$ nas respectivas profundidades de $0-0,10,0,10-0,20$ e $0,20-0,40 \mathrm{~m}$.

Atentando-se para os resultados encontrados referentes aos conteúdos de argila, silte e areia, de acordo com o triângulo da Sociedade Brasileira de Ciência do Solo, vê-se que se trata de um solo com classe textural franco argilo-arenoso para a área em que o solo foi decapitado em 8,60 m, enquanto na sua condição natural a sua classificação corresponde a franco arenoso.

Os solos de textura média apresentam, naturalmente, menor resistência à penetração comparados aos solos argilosos; portanto, os valores de resistência verificados nos tratamentos estudados estavam acima do limite crítico (2,5 MPa), com exceção da camada de 0,00-0,05, indicando degradação da estrutura do solo, mesmo se considerando a textura.

\section{CONCLUSÕES}

1. O sistema com regeneração em que se utilizaram o Pinus e os nichos com regeneração natural, foram os que mais se aproximaram da condição natural de cerrado, no estudo da resistência à penetração do solo.

2. Os efeitos da regeneração do solo, via estudo da resistência à penetração, foram evidenciados na camada de 0,00-0,05 m do solo.

3. Os nichos com regeneração natural apresentaram resistência à penetração baixa na camada de 0,00 a $0,20 \mathrm{~m}$.

4. Na camada de 0,20-0,40 m, com exceção da área natural, as demais apresentaram resistência à penetração alta, o que ocasionaria sérias limitações ao crescimento das raízes.

\section{AGRADECIMENTOS}

Os autores manifestam seu agradecimento ao Professor Dr. Gilson Volpato, da UNESP, pelas orientações para a redação científica; ao Sr. João Josué Barbosa, pelo auxílio na normatização bibliográfica e ao Professor Dr. Paulo C. Ceresini, pela revisão do Abstract.

\section{LITERATURA CITADA}

Alves, M. C. Recuperação do subsolo de um Latossolo Vermelho usado para terrapleno e fundação da usina hidrelétrica de Ilha Solteira, SP. Ilha Solteira: UNESP, 2001. 83p. Tese Livre Docência

Andrade Júnior, R. T. de. Propriedades físico-químicas de um solo em recuperação e adaptação da Brachiaria decumbens, SP. Ilha Solteira: UNESP, 2004. 49p. Trabalho Graduação

Benghough, A. G.; Mullins, C. E. Mechanical impedance to root growth: a review of experimental techniques and root growth responses. Journal Soil Science, Baltimore, v.41, n.3, p.341-58, 1990.

Canarache, A. Penetr - a generalized semi-empirical model estimating soil resistance to penetration. Soil Tillage Research, Amsterdam, v.16, n.1, p.51-70, 1990. 
Carpanezzi, A. A.; Costa, L. G. S.; Kageyama, P. Y.; Castro, C. F. A. Funções múltiplas das florestas. Conservação e recuperação do meio ambiente. In: Congresso Florestal Brasileiro, 6, 1994, Campos de Jordão. Anais... Campos de Jordão: EMBRAPACNPF, 1994, p.216-217.

Cavenage, A. Alterações das propriedades físicas e químicas de um Latossolo Vermelho Escuro sob diferentes usos e manejos. Ilha Solteira: UNESP, 1996. 75p. Trabalho Graduação

CESP - Companhia Energética do Estado de São Paulo Diretoria do Meio Ambiente. Recomposição vegetal. São Paulo: CESP, 1998.11p.

Daniel, L. A.; Carvalho, J. F.; Junqueira, G. S. Avaliação de camadas de solo compactado: Efeito de diferentes sistemas de preparo e cultivo. In: Congresso Brasileiro de Engenharia Agrícola, 24, 1994, Campinas. Anais... Campinas: UNICAMP, 1994, p.300-305.

Demattê, J. L. I. Levantamento detalhado dos solos do "Campus experimental de Ilha Solteira”. Piracicaba: Departamento de Solos, Geologia e Fertilidade ESALQ/USP, 1980. p.11-31.

EMBRAPA - Empresa Brasileira de Pesquisa Agropecuária. Manual de métodos de análise de solo. 2.ed. Rio de Janeiro: EMBRAPA/CNPSO, 1997, p.212.

EMBRAPA - Empresa Brasileira de Pesquisa Agropecuária. Sistema brasileiro de classificação de solos. Rio de Janeiro: Embrapa Solos, 1999, 412p.

EMBRAPA - Empresa Brasileira de Pesquisa Agropecuária. Infraero \& Embrapa Solos recuperam áreas degradadas. http:// www.cnps.embrapa.br/search/pesqs/proj06.html. 15 fev. 2001.
Fornasari Filho, N.; Braga, T. O.; Batistucci, S. G. G.; Montanhesi, M. O. R. Auditoria e sistema de gerenciamento ambiental (ISO 14000). In: Recuperação de áreas degradadas. Simpósio Sul-americano, 1, Simpósio Nacional, 2, 1994, Curitiba. Anais... Curitiba: Fundação de Pesquisas Florestais do Paraná, 1994. p.25-44.

Hakansson, I.; Voorhees, W. B.; Riley, H. Vehide and level factors influencing soil compaction and crop response in different traffic regimes. Soil Tillage Research, Amsterdam, v.11, n.2, p.239-282, 1998.

Klein, V. A.; Libardi, P. L.; Silva, A. P. Resistência mecânica do solo à penetração sob diferentes condições de densidade e teor de água. Engenharia Agrícola, Jaboticabal, v.18, n.2, p.45-54, 1998.

Larson, W. E.; Eynard, A.; Hadas, A.; Lipec, J. Control and avoidance of soil compaction in pratice. In: Soane, B.D. \& Ouwerkerk, C., (ed.) Soil compaction in crop production, 1994. p.597-625.

SAS/STAT. User's Guide. Version G. Cary NC. Fourth Edition. v.1/2, SAS Institute Inc,1990.

Silva, A. M. da; Aguiar, A. V.; Moraes, M. L. T. de; Alves, M. C.; Buzetti, S. Recuperação de áreas degradadas com a utilização de diferentes espécies e variedades de Pinus. Cultura Agronômica, Ilha Solteira, v.11, n.1, p.1-11, 2002.

Souza, Z. M.; Alves, M. C. Movimento de água e resistência à penetração em um Latossolo Vermelho distrófico de cerrado sob diferentes usos e manejos. Revista Brasileira de Engenharia Agrícola e Ambiental, Campina Grande, v.7, n.1, p.18-23, 2003.

Tormena, C. A.; Roloff, G. Dinâmica da resistência à penetração de um solo sob plantio direto. Revista Brasileira de Ciência do Solo, Campinas, v.20, n.2, p.333-339, 1996. 